\title{
Finite-Time Control for Markovian Jump Systems with Polytopic Uncertain Transition Description and Actuator Saturation
}

\author{
Zhongyi Tang ${ }^{1,2}$ \\ ${ }^{1}$ Institute of Automation, Jiangnan University, Wuxi 214122, China \\ ${ }^{2}$ Faculty of Electronic and Engineering, Huaiyin Institute of Technology, Huaian 223003, China \\ Correspondence should be addressed to Zhongyi Tang; 001tzy@163.com
}

Received 24 January 2014; Revised 25 April 2014; Accepted 30 April 2014; Published 20 May 2014

Academic Editor: Shuping He

Copyright (c) 2014 Zhongyi Tang. This is an open access article distributed under the Creative Commons Attribution License, which permits unrestricted use, distribution, and reproduction in any medium, provided the original work is properly cited.

\begin{abstract}
The problem of finite-time $L_{2}-L_{\infty}$ control for Markovian jump systems (MJS) is investigated. The systems considered time-varying delays, actuator saturation, and polytopic uncertain transition description. The purpose of this paper is to design a state feedback controller such that the system is finite-time bounded (FTB) and a prescribed $L_{2}-L_{\infty}$ disturbance attenuation level during a specified time interval is guaranteed. Based on the Lyapunov method, a linear matrix inequality (LMI) optimization problem is formulated to design the delayed feedback controller which satisfies the given attenuation level. Finally, illustrative examples show that the proposed conditions are effective for the design of robust state feedback controller.
\end{abstract}

\section{Introduction}

In the aspect of modeling practical systems with abrupt random changes, such as manufacturing system, telecommunication, and economic systems, MJS have powerful ability. MJS have been extensively studied during the past decades and many systematic results have been obtained [1-3]. The peak-to-peak filtering problem was studied for a class of Markov jump systems with uncertain parameters in [4]. A robust $\mathrm{H}_{2}$ state feedback controller for continuoustime Markov jump linear systems subject to polytopic-type parameter uncertainty was designed in [5]. In [6], the authors address the stabilization problem for single-input Markov jump linear systems via mode-dependent quantized state feedback for control.

Actuator saturation which can lead to poor performance of the closed-loop system is another active research area. In practical situations, it may be encountered sometimes. How to preserve the closed-loop system performance in the case of actuator saturation would be more meaningful. In [7], the $H_{\infty}$ control problem for discrete-time singular Markov jump systems with actuator saturation was considered. In [8] the stochastic stabilization problem for a class of Markov jump linear systems subject to actuator saturation was considered.
In some practical applications, the behavior of the system over a finite-time interval is mainly considered. Finite-time stable (FTS) and Lyapunov asymptotic stability are independent concepts. The concept of FTS was first introduced in [9]. A system is said to be finite-time stable if, given a bound on the initial condition, its state does not exceed a certain threshold during a specified time interval. FTS of linear timevarying systems was considered in [10]. Sufficient conditions for the solvability of both the state and the output feedback problems are stated. Amato [11] provided a necessary and sufficient condition for the FTS of linear-varying systems with jumps. Recently, robust finite-time $H_{\infty}$ control of jump systems was dealt with in [12-14]. In [15], the problems of finite-time stability analysis were investigated for a class of Markovian switching stochastic systems. To the best of authors' knowledge, however, the problem of finite-time $L_{2}-L_{\infty}$ performance for discrete-time MJS with imprecise transition probabilities and time-varying delays has not been well addressed, which motivates our work.

This paper deals with this problem. More specifically, the actuator is saturation. By using the Lyapunov-Krasovskii functional, a new sufficient condition for stochastic asymptotic stability with finite-time $L_{2}-L_{\infty}$ performance is derived in terms of LMI. Based on this, the existence condition of 
the desired performance which guarantees finite-time stability and an $L_{2}-L_{\infty}$ performance of the MJS is presented. A numerical example is provided to show the effectiveness of the proposed results.

Throughout the paper, if not explicitly stated, matrices are assumed to have compatible dimensions. The notation $W>$ $(\geq,<, \leq) 0$ is used to denote a symmetric positive definite (positive semidefinite, negative, negative semidefinite) matrix. $\lambda_{\text {min }}(\cdot)$ and $\lambda_{\max }(\cdot)$ represent the minimum and maximum eigenvalues of the corresponding matrix, respectively. $I$ is the identity matrix with compatible dimensions. $\|\cdot\|$ refers to the Euclidean norm of vectors and $E[\cdot]$ stands for the mathematical expectation. For a symmetric block matrix, "*" is used as an ellipsis for the terms that are obtained by symmetry.

\section{Problem Statement and Preliminaries}

Consider a discrete-time MJS with actuator saturation and delay in the state. Let the system dynamics be described by the following:

$$
\begin{aligned}
x(k+1)= & A_{\theta 1}\left(r_{k}\right) x(k)+A_{\theta 2}\left(r_{k}\right) x(k-d) \\
& +B_{\theta 1}\left(r_{k}\right) \sigma\left(u_{k}\right)+B_{\theta 2}\left(r_{k}\right) w_{k}, \\
z(k)= & C_{\theta 1}\left(r_{k}\right) x(k)+C_{\theta 2}\left(r_{k}\right) x(k-d)+D_{\theta 1}\left(r_{k}\right) w_{k},
\end{aligned}
$$

where $x_{k} \in R^{n}$ is the system state, $z_{k} \in R^{n}$ is the system output, $u_{k} \in R^{m}$ is the control input, $w_{k} \in R^{q}$ is the disturbance input which belongs to $L_{2}[0, \infty)$ and $\sum_{k=0}^{\infty} w_{k}^{T} w_{k}<\kappa^{2}$, and $\kappa$ is a given positive scalar. $A_{\theta 1}\left(r_{k}\right), A_{\theta 2}\left(r_{k}\right), B_{\theta 1}\left(r_{k}\right), B_{\theta 2}\left(r_{k}\right), C_{\theta 1}\left(r_{k}\right), D_{\theta 1}\left(r_{k}\right)$, and $D_{\theta 2}\left(r_{k}\right)$ are appropriately dimensioned real-valued matrices, which belong to the part of convex polyhedron $\Phi\left(r_{k}\right)$ :

$$
\begin{aligned}
& \Phi\left(r_{k}\right) \\
& =\left\{\sum _ { l = 1 } ^ { L } \theta _ { l } \left[A_{l 1}\left(r_{k}\right), A_{l 2}\left(r_{k}\right), B_{l 1}\left(r_{k}\right),\right.\right. \\
& B_{l 2}\left(r_{k}\right), C_{l 1}\left(r_{k}\right), C_{l 2}\left(r_{k}\right), \\
& \left.\left.D_{l 1}\left(r_{k}\right), D_{l 2}\left(r_{k}\right)\right], \sum_{l=1}^{L} \theta_{l}=1, \theta_{l} \geq 0\right\},
\end{aligned}
$$

where $A_{l 1}\left(r_{k}\right), A_{l 2}\left(r_{k}\right), B_{l 1}\left(r_{k}\right), B_{l 2}\left(r_{k}\right), C_{l 1}\left(r_{k}\right), C_{l 2}\left(r_{k}\right)$, and $D_{l 1}\left(r_{k}\right)$ are matrix functions of the random jumping process $\left\{r_{k}\right\}$ (Figure 1), which is a discrete-time Markov chain taking values in a finite set $\Omega=\{1,2, \ldots, S\}$ with transition probabilities:

$$
P\left\{r_{k+1}=j \mid r_{k}=i\right\}=\pi_{i j}
$$

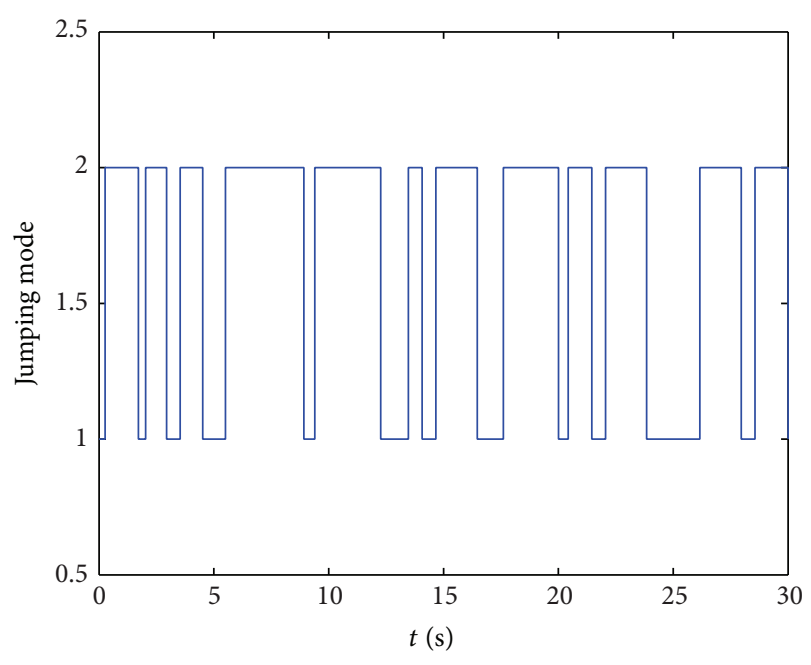

FIGURE 1: Jumping mode.

Here $\pi_{i j} \geq 0$ and for any $i, j \in \Omega, \sum_{j=1}^{s} \pi_{i j}=1$. Assuming that the transition probability $\pi_{i j}$ is not exactly known, a certain range can only be given

$$
\begin{aligned}
& {\left[\pi_{v}(i, 1), \pi_{v}(i, 2), \ldots, \pi_{v}(i, S)\right]} \\
& \quad=\sum_{m=1}^{M} v_{m}\left[\pi_{m}(i, 1), \pi_{m}(i, 2), \ldots, \pi_{m}(i, S)\right],
\end{aligned}
$$

where $v=\left[v_{1} \cdots v_{M}\right]^{T} \in R^{M}$ and $\sum_{m=1}^{M} v_{m}=1$, and the transition probability belongs to the following convex polyhedron:

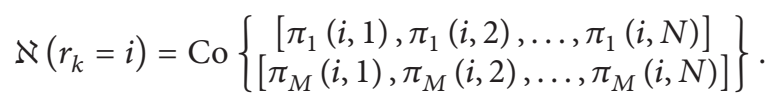

When the system operates in the $i$ th mode $\left(r_{k}=i\right)$, for simplicity, the matrices $A_{\theta 1}\left(r_{k}\right), A_{\theta 2}\left(r_{k}\right), B_{\theta 1}\left(r_{k}\right), B_{\theta 2}\left(r_{k}\right), C_{\theta 1}\left(r_{k}\right)$, and $D_{\theta 1}\left(r_{k}\right)$ are denoted as $A_{\theta 1 i}, A_{\theta 2 i}, B_{\theta 1 i}, B_{\theta 2 i}, C_{\theta 1 i}$, and $D_{\theta 1 i}$, respectively. $d$ is a positive integer denoting the constant delay of the system state (Figures 2 and 3 ).

In system $(1), \sigma(\cdot): R^{m} \rightarrow R^{m}$ is the vector-valued standard saturation function defined as follows:

$$
\sigma(u)=\left[\sigma\left(u_{1}\right), \sigma\left(u_{2}\right), \ldots, \sigma\left(u_{m}\right)\right]^{T},
$$

where $\sigma\left(u_{\theta}\right)=\operatorname{sign}\left(u_{\theta}\right) \min \left\{1,\left|u_{\theta}\right|\right\}$. It is assumed that system (1) is completely controllable. A mode-dependent controller is considered here with the following form:

$$
\sigma(u(k))=\sigma\left(K_{i} x(k)\right),
$$

where $K_{i} \in R^{m \times n}\left(\forall r_{k}=i \in \Omega\right)$ is the controller gain to be determined.

Let $M$ be the set of $m \times m$ diagonal matrices whose diagonal elements are either 1 or 0 . Suppose each element of $M$ is $M_{j}, j=1, \ldots, 2^{m}$, and denote $M_{j}^{-}=I-M_{j}$. Note that $M_{j}^{-}$is also an element of $M$ if $M_{j} \in M$. Let $h_{i j}$ be the $j$ th row of the matrix $H_{i}$, and define the symmetric polyhedron by $\varphi\left(H_{i}\right)=\left\{x(t) \in R^{n}:\left|f_{i j} x(t)\right| \leq 1, i=1,2, \ldots, m\right\}$. 




FIgURE 2: Response of the system state $x_{1}$.

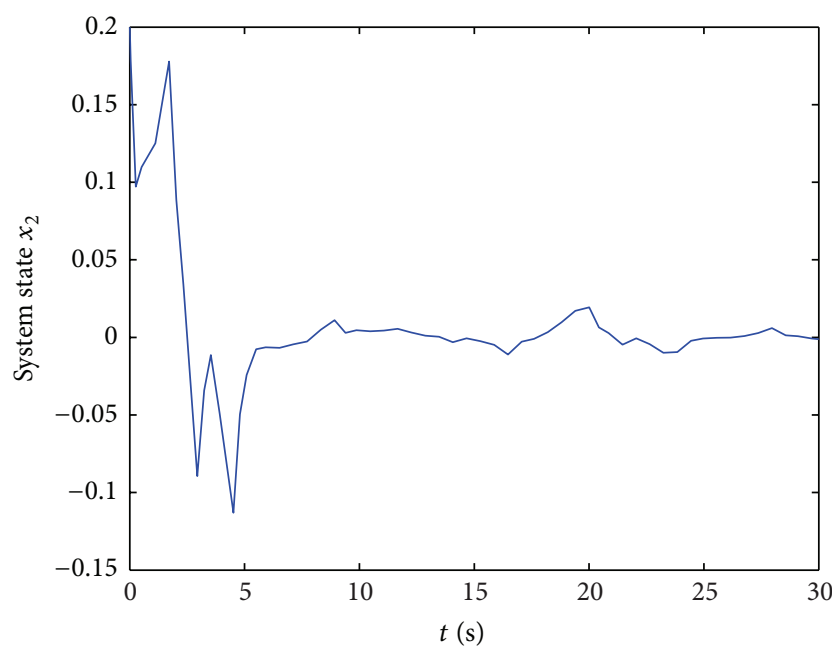

FIgURE 3: Response of the system state $x_{2}$.

Lemma 1 (see [8]). Let $K_{i}, H_{i} \in R^{m \times n}$ be given matrix. For $x(t) \in R^{n}$, if $x(t) \in \varphi\left(H_{i}\right)$, then

$$
\sigma\left(K_{i} x(t)\right)=\sum_{r=1}^{2^{m}} \zeta_{r}\left(M_{r} K_{i}+M_{r}^{-} H_{i}\right) x(t)
$$

where $0 \leq \zeta_{r} \leq 1, \sum_{r=1}^{2^{m}} \zeta_{r}=1$.

By the connection of (6), (7) and (8), the following closedloop MJS are obtained:

$$
\begin{aligned}
x(k+1)= & \left(A_{\theta 1}\left(r_{k}\right)+B_{\theta 1}\left(r_{k}\right)\right. \\
& \left.\times \sum_{r=1}^{2^{m}} \zeta_{r}\left(M_{r} K_{i}+M_{r}^{-} H_{i}\right)\right) x(k) \\
& +A_{\theta 2}\left(r_{k}\right) x(k-d)+B_{\theta 2}\left(r_{k}\right) w_{k} .
\end{aligned}
$$

To describe the main objective of this note more precisely, let us now introduce the following definition for the underlying system.

Definition 2 (see [13]). Given a time constant $T>0$, the MJS

$$
x(k+1)=A_{\theta 1}\left(r_{k}\right) x(k)+A_{\theta 2}\left(r_{k}\right) x(k-d)
$$

are said to be FTS with respect to $\left(\begin{array}{llll}\hbar_{1} & \hbar_{2} & T & R_{i}\end{array}\right)$, if there exist positive matrix $R_{i}>0$, scalars $\hbar_{1}>0$ and $\hbar_{2}>0$, and

$$
\begin{aligned}
E\{ & \left.x^{T}\left(k_{1}\right) R_{i} x\left(k_{1}\right)\right\} \\
& \leq \hbar_{1} \Longrightarrow E\left\{x^{T}\left(k_{2}\right) R_{i} x\left(k_{2}\right)\right\} \\
& \leq \hbar_{2}, \quad k_{1} \in\{-h, \ldots, 0\}, k_{2} \in\{1,2, \ldots, T\} .
\end{aligned}
$$

Definition 3 (see [13]). Given a time constant $T>0$, the MJS

$$
x(k+1)=A_{\theta 1}\left(r_{k}\right) x(k)+A_{\theta 2}\left(r_{k}\right) x(k-d)+B_{\theta 2}\left(r_{k}\right) w_{k}
$$

are said to be finite-time bounded (FTB) with respect to $\left(\begin{array}{llll}\hbar_{1} & \hbar_{2} & T & R_{i}\end{array}\right)$, if there exist positive matrix $R_{i}>0$ and scalars $\hbar_{1}>0$ and $\hbar_{2}>0$, and satisfied (11).

In general, FTB and FTS are different. If there is external disturbance in systems, the concept of FTB is used. Conversely, FTS is addressed.

The objective of this paper is to design a delayed feedback controller which satisfies the given attenuation level of system (1). The design procedure is given in the next section.

Definition 4. The time-delay MJS (1) is said to be finitetime $L_{2}-L_{\infty}$ control with respect to $\left(\hbar_{1} \hbar_{2} \quad T \quad R_{i}\right)$ and performance $\gamma$, where $R_{i}>0, \gamma>0, \hbar_{1}>0$, and $\hbar_{2}>0$, if the time-delay MJS (1) is stochastically FTB and under the zero-initial condition the output $z(k)$ satisfies

$$
\|z(k)\|_{\infty}<\gamma\|w(k)\|_{2}
$$

for all nonzero $w(k) \in L_{2}[0, \infty)$ subject to the zero-initial condition.

\section{Main Results}

In this section, firstly stochastic FTB analysis of nominal time-delay MJS (1) is provided. Then, these results will be extended to the MJS (1) with actuator saturation and uncertain transition probability. LMI conditions are established.

Lemma 5. System (1) with $\sigma\left(u_{k}\right) \equiv 0$ is stochastic FTB with respect to $\left(\hbar_{1} \hbar_{2} d R_{i} N\right)$; if for scalars $\varsigma \geq 1, \hbar_{1}>0$, and $\hbar_{2}>0$, there exist symmetric matrices $R_{i}>0(i \in \Omega)$ 
and $Q_{i}>0(i \in \Omega)$, such that the following matrix inequalities hold:

$$
\begin{aligned}
\Lambda & =\left[\begin{array}{ccc}
A_{l 1 i}^{T} \bar{P}_{i} A_{l 1 i}-P_{i}+Q & * & * \\
A_{l 2 i}^{T} \bar{P}_{i} A_{l 1 i} & -Q+A_{l 2 i}^{T} \bar{P}_{i} A_{l 2 i} & * \\
B_{l 2 i}^{T} \bar{P}_{i} A_{l 1 i} & B_{l 2 i}^{T} \bar{P}_{i} A_{l 2 i} & B_{l 2 i}^{T} \bar{P}_{i} B_{l 2 i}-I
\end{array}\right] \\
& <0
\end{aligned}
$$

$$
\varsigma^{k}\left[\lambda_{\max }\left(\widetilde{P}_{r(0)}\right)+\lambda_{\max }(Q) \cdot d\right] c_{1} \leq c_{2} \cdot \lambda_{\min }\left(\widetilde{P}_{i}\right)
$$

where $\bar{P}_{i}=\sum_{j=1}^{S} \pi_{v i j} P_{j}$.
Proof. Choose the following Lyapunov functional:

$$
V(k)=x^{T}(k) P_{i} x(k)+\sum_{n=k-d}^{k-1} x^{T}(n) Q x(n) .
$$

The proof of Lemma 5 is divided into two parts. In the first part, the following inequality is obtained:

$$
E\{V(k)\}<\varsigma^{k} E\{V(0)\}+\varsigma^{k} w^{T}(k) w(k) .
$$

Then, we compute

$$
\begin{aligned}
\Delta V(k) & =E\{V(k+1)\}-V(k) \\
& =\sum_{j=1}^{S} \pi_{v i j} x^{T}(k+1) P_{j} x(k+1)-x^{T}(k) P_{i} x(k)+\sum_{j=1}^{S} \pi_{v i j} x^{T}(k) Q x(k)-x^{T}(k-d) Q x(k-d) \\
& =\vartheta^{T}(k)\left[\begin{array}{ccc}
A_{\theta 1 i}^{T} \bar{P}_{i} A_{\theta 1 i}-P_{i}+Q & * & * \\
A_{\theta 2 i}^{T} \bar{P}_{i} A_{\theta 1 i} & -Q+A_{\theta 2 i}^{T} \bar{P}_{i} A_{\theta 2 i} & * \\
B_{\theta 2 i}^{T} \bar{P}_{i} A_{\theta 1 i} & B_{\theta 2 i}^{T} \bar{P}_{i} A_{\theta 2 i} & B_{\theta 2 i}^{T} \bar{P}_{i} B_{\theta 2 i}
\end{array}\right] \vartheta(k) \\
& \left.=\vartheta^{T}(k)\left[\begin{array}{ccc}
A_{l 1 i}^{T} \bar{P}_{i} A_{l 1 i}-P_{i}+Q & * \\
A_{l 2 i}^{T} \bar{P}_{i} A_{l 1 i} & -Q+A_{l 2 i}^{T} \bar{P}_{i} A_{l 2 i} & * \\
B_{l 2 i}^{T} \bar{P}_{i} A_{l 1 i} & B_{l 2 i}^{T} \bar{P}_{i} A_{l 2 i} & B_{l 2 i}^{T} \bar{P}_{i} B_{l 2 i}
\end{array}\right]\right]
\end{aligned}
$$

where $\vartheta(k)=[x(k) \quad x(k-d) \quad w(k)]$.

Note condition (14); it follows that

$$
\begin{aligned}
E\{V(k+1)\}-V(k)< & (\varsigma-1) V(k) \\
& +w^{T}(k) w(k), \quad \varsigma \geq 1 .
\end{aligned}
$$

Therefore, we obtain that

$$
E\{V(k+1)\}<\varsigma V(k)+w^{T}(k) w(k) .
$$

That is,

$$
\begin{array}{ccc}
E\{V(x(1), r(1))\} & < & \varsigma V(x(0), r(0))+w^{T}(k) w(k) \\
\vdots & \vdots & \vdots \\
E\{V(x(k+1), r(k+1))\} & <\varsigma E\{V(x(k), r(k))\}+w^{T}(k) w(k) .
\end{array}
$$

By recursive,

$$
E\{V(k)\}<\varsigma^{k} E\{V(0)\}+E\left\{\sum_{\tau=0}^{k-1} \varsigma^{k-\tau-1} w^{T}(\tau) w(\tau)\right\} .
$$

Then the inequality in (17) is obtained.
In the second part, stochastic FTB is established:

$$
\begin{aligned}
E\{V(k)\} & =E\left\{x^{T}(k) P_{i} x(k)+\sum_{n=k-d}^{k-1} x^{T}(n) Q x(n)\right\} \\
& \geq \lambda_{\min }\left(\widetilde{P}_{i}\right) E\left\{x^{T}(k) R x(k)\right\} .
\end{aligned}
$$

On the other hand,

$$
\begin{aligned}
\varsigma^{k} E\{V(0)\} \leq \varsigma^{k}[ & \lambda_{\max }\left(\widetilde{P}_{r(0)}\right) E\left\{x^{T}(0) R x(0)\right\} \\
& \left.+\lambda_{\max }(Q) E\left\{\sum_{n=-d}^{-1} x^{T}(n) R x(n)\right\}\right] .
\end{aligned}
$$

From Definition 2, we have

$$
\varsigma^{k} E\{V(0)\} \leq \varsigma^{k}\left\{\left[\lambda_{\max }\left(\widetilde{P}_{r(0)}\right)+\lambda_{\max }(Q) \cdot d\right]\right\} \hbar_{1}+\varsigma^{k} .
$$


By (23) and (25), we know

$$
\begin{aligned}
& E\left\{x^{T}(k) R x(k)\right\} \\
& \leq \frac{\varsigma^{k}\left\{\left[\lambda_{\max }\left(\widetilde{P}_{r(0)}\right)+\lambda_{\max }(Q) \cdot d\right]+1\right\} \hbar_{1}}{\lambda_{\min }\left(\widetilde{P}_{i}\right)} \\
& \leq \hbar_{2} .
\end{aligned}
$$

This completes the proof.

Theorem 6. System (1) is finite-time $L_{2}-L_{\infty}$ control and satisfies the given lever $\gamma$ with respect to $\left(\hbar_{1} \hbar_{2} d R_{i} N\right)$; if for scalars $\varsigma \geq 1, \hbar_{1}>0$, and $\hbar_{2}>0$, there exist symmetric matrices $R_{i}>0(i \in \Omega)$ and $Q>0$, such that the following matrix inequalities hold:

$\Theta_{1}$

$$
\begin{aligned}
& =\left[\begin{array}{ccc}
A_{l 1 i}^{T} \bar{P}_{i} A_{l 1 i}-P_{i}+Q & * & * \\
A_{l 2 i}^{T} \bar{P}_{i} A_{l 1 i} & -Q+A_{l 2 i}^{T} \bar{P}_{i} A_{l 2 i} & * \\
B_{l 2 i}^{T} \bar{P}_{i} A_{l 1 i} & B_{l 2 i}^{T} \bar{P}_{i} A_{l 2 i} & B_{l 2 i}^{T} \bar{P}_{i} B_{l 2 i}-I
\end{array}\right] \\
& <0,
\end{aligned}
$$

$$
\begin{gathered}
\Theta_{2}=\left[\begin{array}{cccc}
-P_{i} & * & * & * \\
0 & -Q & * & * \\
0 & 0 & -I & * \\
C_{l 1 k} & C_{l 2 k} & D_{l 2 k} & -\gamma^{2} I
\end{array}\right] \\
\varsigma^{k}\left[\lambda_{\max }\left(\widetilde{P}_{r(0)}\right)+\lambda_{\max }(Q) \cdot d\right] c_{1} \leq c_{2} \cdot \lambda_{\min }\left(\widetilde{P}_{i}\right) .
\end{gathered}
$$

Proof. System (1) with $\sigma\left(u_{k}\right) \equiv 0$ is FTB according to Lemma 5 and inequality (27).

Subsequently, to establish the energy-to-peak performance for the system (1), assume that the initial values for the plant are zeros and consider the following function:

$$
\aleph:=E\{V(k)\}-\sum_{i=0}^{k-1} w_{i}^{T} w_{i}
$$

For any nonzero $w_{k} \in l_{2}[0, \infty)$ and $k>0$, it follows from (18) that

$$
\begin{aligned}
\aleph & :=E\left\{\sum_{i=0}^{k-1} \Delta V(i)-\sum_{i=0}^{k-1} w_{i}^{T} w_{i}\right\} \\
& =\vartheta^{T}(k) \Theta_{1} \vartheta(k) .
\end{aligned}
$$

It follows from (27) that $E\{V(k)\}<\sum_{i=0}^{k-1} w_{i}^{T} w_{i}$.
For all the time instants $k>0$, the expectation of the output can be evaluated as

$$
\begin{aligned}
E\left\{z_{k}^{T} z_{k}\right\}= & E\left\{\vartheta^{T}(k)\left[\begin{array}{lll}
C_{\theta 1 k} & C_{\theta 2 k} & D_{\theta 1 k}
\end{array}\right]^{T}\right. \\
& \left.\times\left[\begin{array}{lll}
C_{\theta 1 k} & C_{\theta 2 k} & D_{\theta 1 k}
\end{array}\right] \vartheta(K)\right\} \\
< & E\left\{\gamma^{2} \vartheta^{T}(k)\left[\begin{array}{ccc}
P_{i} & 0 & 0 \\
* & \bar{Q}_{i} & 0 \\
* & * & I
\end{array}\right] \vartheta(k)\right\} \\
< & \gamma^{2} E\left\{\sum_{i=0}^{k} w_{i}^{T} w_{i}\right\}<\gamma^{2} E\left\{\sum_{i=0}^{\infty} w_{i}^{T} w_{i}\right\} \\
= & \gamma^{2}\|w\|_{2}^{2} .
\end{aligned}
$$

Applying Definition 4, the statement of Theorem 6 is true.

Theorem 7. Consider the uncertain time-delay system (1); there exists a state feedback controller $\sigma\left(K_{i} x(t)\right)$ such that the uncertain time-delay system (1) is finite-time $L_{2}-L_{\infty}$ control with respect to $\left(\hbar_{1} \hbar_{2}\right.$ d $\left.R_{i} N\right)$, if the following LMIs hold:

$$
\begin{aligned}
\Lambda_{1} & =\left[\begin{array}{ccccccc}
-X_{i} & 0 & 0 & \varepsilon_{14} & \cdots & \varepsilon_{16} & X_{i} \\
* & -R & 0 & \varepsilon_{24} & \cdots & \varepsilon_{26} & 0 \\
* & * & -I & \varepsilon_{34} & \cdots & \varepsilon_{36} & 0 \\
* & * & * & \varepsilon_{44} & \cdots & 0 & 0 \\
* & * & * & * & \ddots & 0 & 0 \\
* & * & * & * & * & \varepsilon_{66} & 0 \\
* & * & * & * & * & * & -R
\end{array}\right]<0, \\
\Lambda_{2} & =\left[\begin{array}{ccccc}
-P_{i} & * & * & * \\
0 & -Q & * & * \\
0 & 0 & -I & * \\
C_{l 1 k} & C_{l 2 k} & D_{l 2 k} & -\gamma^{2} I
\end{array}\right]<0,
\end{aligned}
$$

$$
\varsigma^{k}\left[\lambda_{\max }\left(\widetilde{P}_{r(0)}\right)+\lambda_{\max }(Q) \cdot d\right] c_{1} \leq c_{2} \cdot \lambda_{\min }\left(\widetilde{P}_{i}\right)
$$

where $\varepsilon_{14}=\sqrt{\pi_{i 1}}\left(A_{l 1 i}+B_{l 1 i}\left(M_{r} Y_{i}+M_{r}^{-} Z_{i}\right), \varepsilon_{24}=\sqrt{\pi_{i 1}} R A_{l 2 i}\right.$, $\varepsilon_{34}=\sqrt{\pi_{i 1}} R B_{l 2 i}, \varepsilon_{44}=-X_{1}, \varepsilon_{16}=\sqrt{\pi_{i S}}\left(A_{l 1 i}+B_{l 1 i}\left(M_{r} Y_{i}+\right.\right.$ $\left.\left.M_{r}^{-} Z_{i}\right)_{i}\right), \varepsilon_{26}=\sqrt{\pi_{i S}} R A_{l 2 i}, \varepsilon_{36}=\sqrt{\pi_{i S}} R B_{l 2 i}$, and $\varepsilon_{66}=-X_{S}$.

The state feedback controller is designed as $\sigma\left(K_{i} x(t)\right)=$ $\sum_{r=1}^{2^{m}} \zeta_{r}\left(M_{r} K_{i}+M_{r}^{-} H_{i}\right) x(t)$.

Proof. Noting condition (27) and $\bar{P}_{i}=\Gamma_{i} \kappa \Gamma_{i}^{T}$, where $\kappa=$ $\operatorname{diag}\left\{P_{1}, \ldots, P_{S}\right\}, \Gamma_{i}=\left[\sqrt{p_{i 1}} I, \ldots, \sqrt{p_{i S}} I\right]$ thus $\Theta_{1}$ can be rewritten as

$$
\begin{aligned}
\Theta_{1}= & {\left[\begin{array}{ccc}
-P_{i}+Q & * & * \\
0 & -Q & * \\
0 & 0 & -I
\end{array}\right] } \\
& +\left[\begin{array}{c}
A_{l 1 i}^{T} \\
A_{l 2 i}^{T} \\
B_{l 2 i}^{T}
\end{array}\right] \kappa\left[\begin{array}{lll}
A_{l 1 i} & A_{l 2 i} & B_{l 2 i}
\end{array}\right]<0 .
\end{aligned}
$$


Using Schur complement, it can be obtained

$$
\left[\begin{array}{cccc}
-P+Q & 0 & 0 & A_{l 1 i}^{T} \Gamma_{i} \\
* & -Q & 0 & A_{l 2 i}^{T} \Gamma_{i} \\
* & * & -I & B_{l 2 i}^{T} \Gamma_{i} \\
* & * & * & -\kappa^{-1}
\end{array}\right]<0 .
$$

Let $X_{i}=P_{i}^{-1}, R=Q^{-1}, Y_{i}=K_{i} X_{i}$, and $Z_{i}=H_{i} X_{i}$. Preand postmultiplying (35) by $\operatorname{diag}\left\{X_{i}, R, I, I\right\}$ and then using Schur complement, then inequality (32) is obtained. Implying Theorem 6, we can conclude that the corresponding closedloop system is finite-time $L_{2}-L_{\infty}$ control. This completes the proof.

\section{Numeral Example}

To illustrate the proposed results, a numerical example is considered for finite-time $L_{2}-L_{\infty}$ control. The system is described by (1) and assumed to have two modes; $\Omega=\{1,2\}$. The mode switching is governed by a Markov chain that has the following transition probability matrix:

$$
P=\left[\begin{array}{ll}
0.2 & 0.8 \\
0.4 & 0.6
\end{array}\right] .
$$

The system matrices are as follows:

$$
\begin{gathered}
A_{111}=A_{211}=\left[\begin{array}{cc}
0.3 & 0.102 \\
-0.663 & 0.3
\end{array}\right], \\
A_{112}=A_{212}=\left[\begin{array}{cc}
0.8 & 0.0539 \\
-0.8655 & 0.8
\end{array}\right], \\
A_{121}=A_{221}=\left[\begin{array}{cc}
0.5 & 0.06 \\
-0.843 & 0.5
\end{array}\right], \\
A_{122}=A_{222}=\left[\begin{array}{cc}
0.9 & 0.0766 \\
-0.7661 & 0.9
\end{array}\right], \\
B_{111}=B_{211}=\left[\begin{array}{ll}
0.0005 \\
0.0539
\end{array}\right], \\
B_{212}=B_{112}=\left[\begin{array}{c}
0.005 \\
0.1078
\end{array}\right], \\
B_{121}=B_{221}=\left[\begin{array}{ll}
0.0045 \\
0.0539
\end{array}\right], \\
C_{111}=C_{211}=C_{112}=C_{221}=C_{122}=C_{222}=[0.30], \\
D_{111}=D_{211}=D_{112}=D_{212}=0.3 . \\
B_{122}=B_{222}=\left[\begin{array}{ll}
0.0045 \\
0.1078
\end{array}\right],
\end{gathered}
$$

Assume $L_{2}-L_{\infty}$ performance of level $\gamma=0.3$; by applying Theorem 7, we can explicitly compute the optimally achievable closed-loop $L_{2}-L_{\infty}$ performance $\gamma$ from Theorem 7 as $\gamma=0.2056$. Response of the system state is depicted in Figures 2 and 3 .

\section{Conclusion}

The problem of finite-time $L_{2}-L_{\infty}$ control for MJS has been studied. By using the Lyapunov functional approach, a sufficient condition is derived such that the closed-loop MJS are stochastic FTB and satisfy the given level. The controller can be obtained by using the exiting LMI optimization techniques. Finally, numerical and simulation results demonstrate the effectiveness of the results of the paper.

\section{Conflict of Interests}

The author declares that there is no conflict of interests regarding the publication of this paper.

\section{Acknowledgment}

This work was supported by the National Natural Science Foundation of China under Grant no. 61273087.

\section{References}

[1] H. Shen, S. Xu, J. Lu, and J. Zhou, "Passivity-based control for uncertain stochastic jumping systems with mode-dependent round-trip time delays," Journal of the Franklin Institute, vol. 349, no. 5, pp. 1665-1680, 2012.

[2] H. Shen, S. Xu, J. Zhou, and J. Lu, "Fuzzy $H_{\infty}$ filtering for nonlinear Markovian jump neutral systems," International Journal of Systems Science, vol. 42, no. 5, pp. 767-780, 2011.

[3] Z.-G. Wu, P. Shi, H. Su, and J. Chu, "Asynchronous $l_{2}-l_{\infty}$ filtering for discrete-time stochastic Markov jump systems with randomly occurred sensor nonlinearities," Automatica, vol. 50, no. 1, pp. 180-186, 2014.

[4] S. He and F. Liu, "Robust peak-to-peak filtering for Markov jump systems," Signal Processing, vol. 90, no. 2, pp. 513-522, 2010.

[5] J. Dong and G.-H. Yang, "Robust $H_{2}$ control of continuous-time Markov jump linear systems," Automatica, vol. 44, no. 5, pp. 1431-1436, 2008.

[6] N. Xiao, L. Xie, and M. Fu, "Stabilization of Markov jump linear systems using quantized state feedback," Automatica, vol. 46, no. 10, pp. 1696-1702, 2010.

[7] S. Ma and C. Zhang, " $H_{\infty}$ control for discrete-time singular Markov jump systems subject to actuator saturation," Journal of the Franklin Institute, vol. 349, no. 3, pp. 1011-1029, 2012.

[8] H. Liu, E.-K. Boukas, F. Sun, and D. W. C. Ho, "Controller design for Markov jumping systems subject to actuator saturation," Automatica, vol. 42, no. 3, pp. 459-465, 2006.

[9] G. V. Kamenkov, "On stability of motion over a finite interval of time," Journal of Applied Mathematics and Mechanics, vol. 17, pp. 529-540, 1953.

[10] F. Amato, M. Ariola, and C. Cosentino, "Finite-time stability of linear time-varying systems: analysis and controller design," IEEE Transactions on Automatic Control, vol. 55, no. 4, pp. 10031008, 2010. 
[11] F. Amato, R. Ambrosino, M. Ariola, and C. Cosentino, "Finitetime stability of linear time-varying systems with jumps," Automatica, vol. 45, no. 5, pp. 1354-1358, 2009.

[12] Y. Zhang, C. Liu, and H. Sun, "Robust finite-time $H_{\infty}$ control for uncertain discrete jump systems with time delay," Applied Mathematics and Computation, vol. 219, no. 5, pp. 2465-2477, 2012.

[13] S. He and F. Liu, "Finite-time $H_{\infty}$ fuzzy control of nonlinear jump systems with time delays via dynamic observer-based state feedback," IEEE Transactions on Fuzzy Systems, vol. 20, pp. 605614, 2012.

[14] Y. Zhang and C. Liu, "Observer-based finite-time $H_{\infty}$ control of discrete-time Markovian jump systems," Applied Mathematical Modelling, vol. 37, no. 6, pp. 3748-3760, 2013.

[15] Y. Yang, J. Li, and G. Chen, "Finite-time stability and stabilization of Markovian switching stochastic systems with impulsive effects," Journal of Systems Engineering and Electronics, vol. 21, no. 2, pp. 254-260, 2010. 


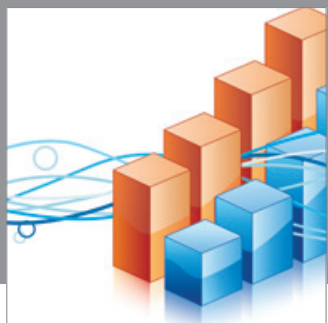

Advances in

Operations Research

mansans

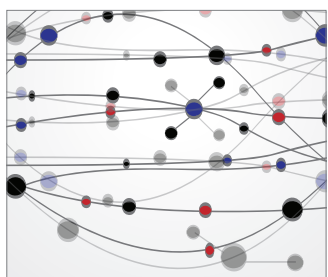

The Scientific World Journal
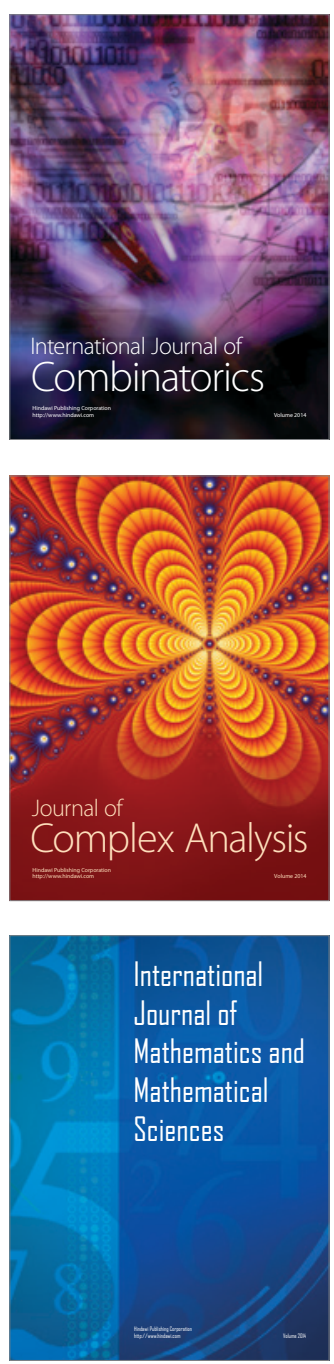
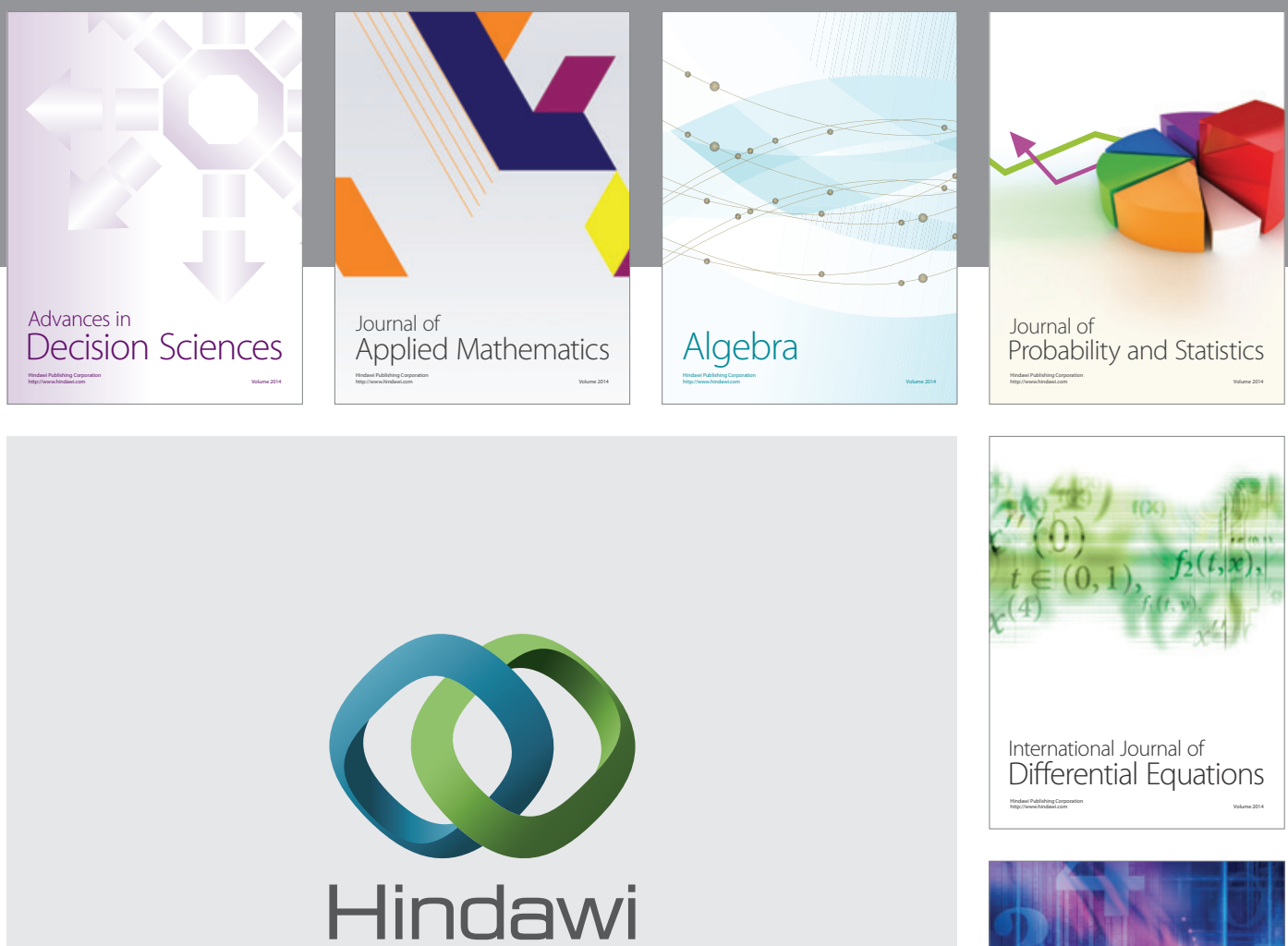

Submit your manuscripts at http://www.hindawi.com
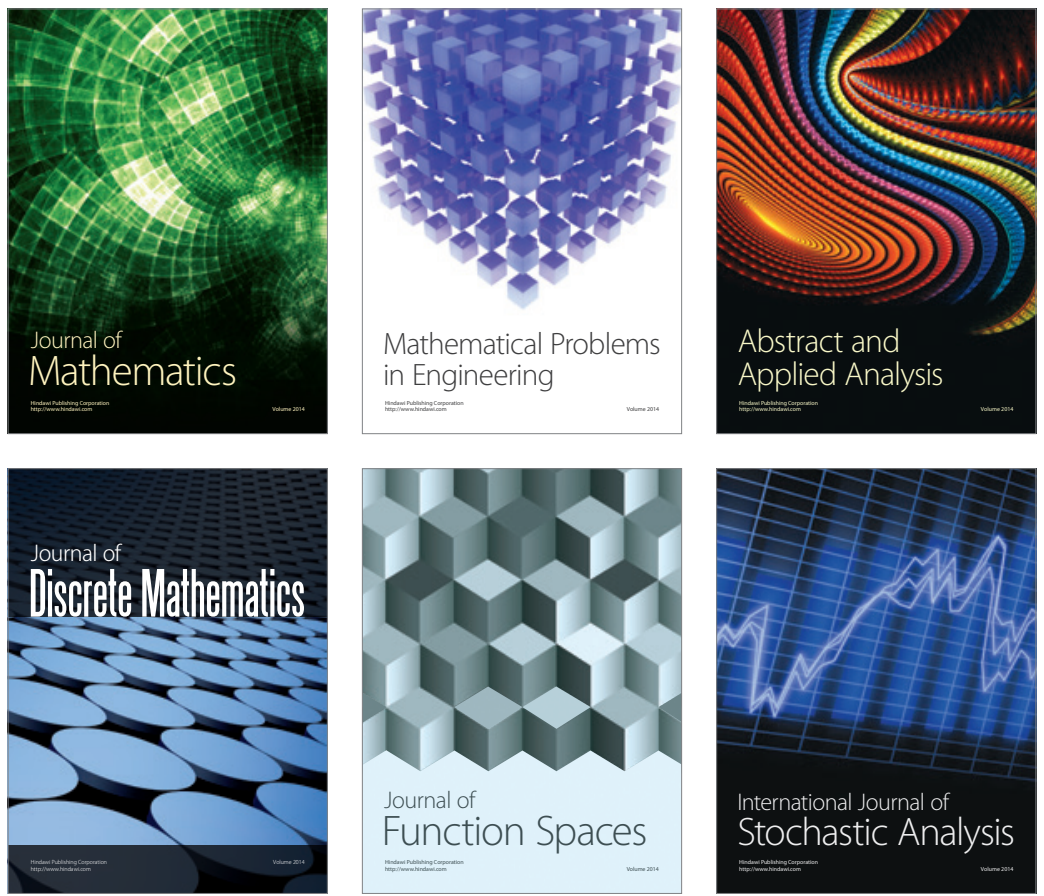

Journal of

Function Spaces

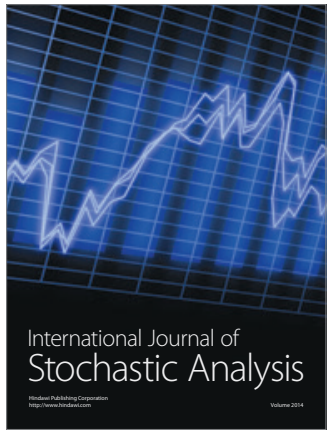

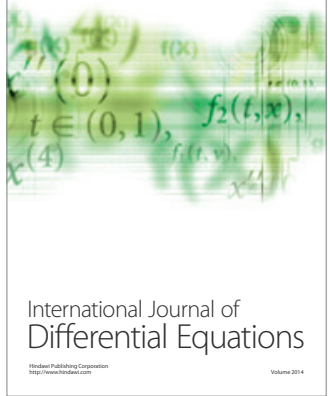
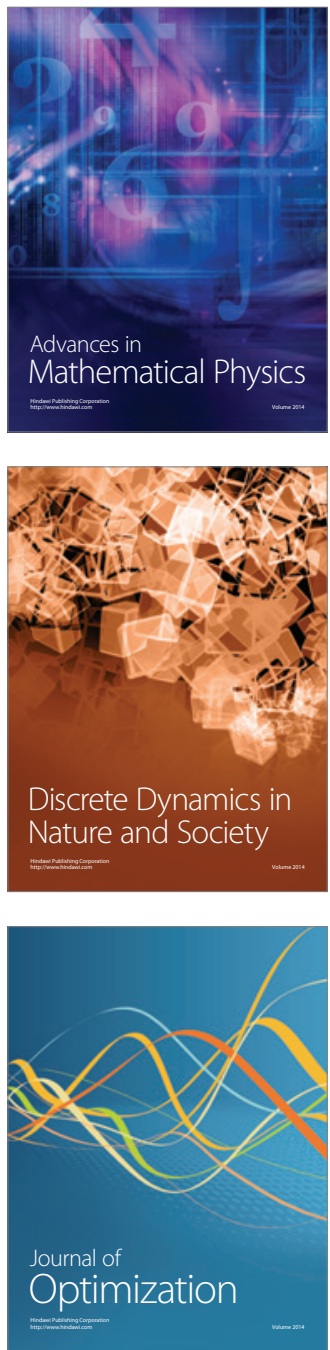